\title{
IMAGE RESTORATION BY IMPROVED ITERATIVE SHRINKAGE THRESHOLDING ALGORITHM
}

\author{
A. K. Kumaresh \\ Associate Professor, Department of Computer Science \\ The M. D. T. Hindu College Tirunelveli
}

\begin{abstract}
The problem of restoration of digital images plays a central role in multitude important applications. A particularly challenging instance of this problem occurs in the case when the degradation phenomenon is modelled by ill-conditional operator. In such situation, the presence of noise makes it impossible to recover a valuable approximation of the image of interest without using some priori information called as simply priors is essential for image restoration, rendering it stable and robust to noise. Particularly, if the original image is known to be a piecewise smooth function, a total variation (TV) based image restoration can be applied. This paper proposes an algorithm for unconstrained optimization problem where the objective function includes a data fidelity term and a nonsmooth regulaizer.Total Variation method is employed to find solution of the problem based on the Improved Iterative Shrinkage Thresholding Algorithm (IISTA). IISTA is performed through a recursive application of two simple procedures linear filtering and soft thresholding. An experimental result shows that proposed algorithm performs well when compared with the existing methods.
\end{abstract}

Keywords: ISTA, IISTA, 12 data fidelity term, total variation, image restoration, inverse problems.

Cite this Article: A. K. Kumaresh, Image Restoration by Improved Iterative Shrinkage Thresholding Algorithm, International Journal of Computer Engineering and Technology, 10(2), 2019, pp. 91-98.

http://iaeme.com/Home/issue/IJCET?Volume=10\&Issue=2

\section{INTRODUCTION}

Image restoration is one of the classical linear inverse problems in imaging [1] [2] [3], since 1960. In this class of problems, a noisy indirect observation $\mathbf{y}$ of the original image $\mathbf{x}$ is modeled as

$$
\mathrm{y}=\mathrm{Bx}+\mathrm{n}
$$


where $\mathbf{B}$ is the matrix representation of the direct operator and $\mathbf{n}$ is the noise. Vector notation for images, where the pixels on an $\mathrm{MXN}$ images are stacked into an $(\mathrm{MN})$-vector in lexicographic order. If $\mathrm{n}$ denotes the number of elements of $\mathbf{x}$, thus, $\mathrm{x} \in \mathrm{R}^{\mathrm{n}}$, while $\mathrm{y} \in \mathrm{R}^{\mathrm{m}}$ ( $\mathrm{m}$ and $\mathrm{n}$ may or may not be equal). Note that $\mathrm{B}$ may be the matrix representation of convolution of the direct operator for image deblurring or deconvolution. If this convolution is periodic, $\mathbf{B}$ is then a block circulant matrix. B may be linear direct operator such as a set of tomographic projections (Radon Transform), a partially observed transform e.g., Fourier Transform or loss of part of the image pixels. Several approaches are available to overcome Image Restoration problem.

Section 2 discusses some of the important methods. Section 3 highlights the proposed IISTA. Section 4 analyses the experimental results and section 5 concludes the paper.

\section{EXISTING IMAGE RESTORATION APPROACHES}

One of the emerging techniques of Image Restoration is Total Variation. The Total Variation (TV) has been introduced Rudin, Osher and Fatemi (ROF) as a regularizing criterion for solving inverse problems. It has proved to be quite efficient for regularizing images without smoothing the boundaries of the object. One of the key difficulties in the TV based deblurring problem is the presence of nonsmooth TV norm in its objective. It has proved that the matrix vector method requires lower memory with slower convergence. The objective of this paper to discuss very simple and fast algorithms for constrained TV based image denoising and deblurring problems.

The standard algorithm for solving problem of the form (1) is so called Iterative Shrinkage Thresholding (IST). IST can be derived as an Expectation-Maximization (EM) algorithm [4], as a Majorization-Minimization (MM) [5] method [6][7] or as a Forward-Backward Splitting technique [8][9].

The IST or Moreau proximity mapping [8] or the denoising function associated with regularizer $\phi$ which provides the solution of the corresponding pure denoising problem.

\subsection{SYNTHESIS APPROACH}

Let the unknown image $\mathrm{x}$ be represented as a linear combination of the elements of some frame i.e., $\mathbf{x}=\mathbf{w} \boldsymbol{\beta}$ where $\beta \in \mathrm{R}^{\mathrm{d}}$ and the column of the $\mathrm{n} \mathbf{x} \mathrm{d}$ matrix $\mathbf{w}$ are the elements of the wavelet frame. Then the coefficients of this representations are estimated from the noisy image, under of the well-known sparsity inducing regularizers, such as $1_{1}$ norm [6] .Formally, this leads to the following optimization problem

$$
\boldsymbol{\beta}=\arg _{\boldsymbol{\beta}}^{\min } \frac{1}{2}\|B W \boldsymbol{\beta}-\boldsymbol{y}\|_{2}^{2}+\tau \phi(\beta)
$$

Where $\phi: \mathrm{Rd} \rightarrow \mathrm{R}$ usually called the regualizer or regularization function is often non smooth, or may be even nonconvex and $\tau \geq 0$ is the regularization parameter. This formulation is called synthesis approach [10].

\subsection{ANALYSISAPPROACH}

An alternative formulation applies a regulaizer directly to the unknown image, leading to criteria of the form

$$
\mathbf{x}=\arg _{\mathbf{x}} \min _{\frac{1}{2}}\|\boldsymbol{B} \mathbf{x}-\boldsymbol{y}\|_{2}^{2}+\tau \phi(\mathbf{x})
$$

Where $\phi: \mathrm{R}^{\mathrm{n}} \rightarrow \mathrm{R}$ is the regulaizer. This type of criteria are called analysis approach, since they are based on a regulaizer that analysis the image itself. The best known regulazier is the total variation (TV) norm [11] [12].

The matrix $\mathbf{B}$ given in equation (2) and (3) has been found that it is a block circulant matrix. Then the multiplication of the form $\mathbf{W v}$ or $\mathbf{W}^{\mathbf{H}} \mathbf{v}$ can be performed by Fast Fourier Transform 
(FFT) algorithm, where $\mathbf{w}$ is a matrix and $\mathbf{v}$ is a vector. The equation (2) and (3) can be written in common form

$$
\mathbf{x}=\arg \min _{\mathbf{x}}{ }_{2}^{1}\|A \mathbf{x}-y\|_{2}^{2}+\tau \phi(\mathbf{x})
$$

Where $\mathbf{A}=\mathbf{B W}$ or $\mathbf{A}=\mathbf{B}$.

\subsection{The General Optimization model}

$$
\text { Given } \min \{\mathrm{F}(\mathbf{x})=\mathrm{f}(\mathbf{x})+\mathrm{g}(\mathbf{x}): \mathbf{x} \in \mathrm{E}
$$

with the following assumptions.

1. The vector space $\mathrm{E}$ stands for a finite dimensional Euclidean space with inner product $<,>>$ and norm $\left.\|\cdot\|=<^{\prime},\right\rangle^{1 / 2}$.

2. $\mathrm{g}: \mathrm{E} \rightarrow(-\infty,+\infty]$ is a proper closed convex function.

3. $\mathrm{f}: \mathrm{E} \rightarrow \mathrm{R}$ is a continuously differentiable with Lipschitz continuous gradient

$\mathrm{L}(\mathrm{f})$

$\|\nabla f(x)-\nabla f(y)\|=L(f)\|\mathbf{x}-\mathbf{y}\|$ for every $x, y \in E$ where $\|$.$\| denotes standard Euclidean norm$ and $\mathrm{L}(\mathrm{f})>0$ is the Lipschitz constant $[8]$ of $\nabla f$.

4. Equation (5) is solvable $X_{*}=\operatorname{argmin} \mathrm{F} \neq 0$ and for $\mathbf{x}^{*} \in \mathrm{X}$, we $\operatorname{set} \boldsymbol{F}_{*}=\boldsymbol{F}\left(\boldsymbol{x}^{*}\right)$. In particular, the standard convex constrained minimization problem:

$$
\operatorname{Min}\{\mathrm{f}(\mathrm{x}): \mathbf{x} \in \mathrm{C}\}
$$

Is obtained by choosing $\mathrm{g}(\mathbf{x})=\delta_{\mathrm{c}}(\mathbf{x})$, with $\mathrm{C} \subseteq \mathrm{E}$ some closed convex set and $\delta_{\mathrm{c}}$ being the indicator function on C. Likewise, with $\mathrm{f}(\mathbf{x}) \equiv 0$, the general non smooth convex minimization problem is obtained [13].

\subsection{Proximal Map}

The key role within approach is the proximal map of Moreau associated to a convex function.

Given proper closed convex function; $\mathrm{E} \rightarrow(-\infty,+\infty]$ and any scalar $\mathrm{t}>0$, the proximal map associated to $\mathrm{g}$ is defined by

$$
\operatorname{prox}_{t}(g)(\mathbf{x})=\arg \min _{x}\left\{g(\mathbf{u})+\frac{1}{2 t}\|\mathbf{u}-\mathbf{x}\|^{2}\right\}
$$

The proximal map associated with a closed proper convex function $g$ has the following properties.

Lemma Let $\mathrm{g} \rightarrow(-\infty,+\infty]$ be a closed proper convex function and for any $\mathrm{t}>0$,

$$
\text { Let } g_{t}(x)={ }_{x}^{\inf }\left\{g(\mathbf{u})+\frac{1}{2 t}\|\mathbf{u}-\mathbf{x}\|^{2}\right\}
$$

Then, a) The infirmum in (8) is attained at the unique point $\operatorname{prox}_{\mathrm{t}}(\mathrm{g})(\mathbf{x})$. As a consequence, the map $(I+\partial g)^{-1}$ is single valued from $E$ into itself and

$$
(I+\partial g)^{-1}(\mathbf{x}) \equiv \operatorname{prox}_{\mathrm{t}}(\mathrm{g})(\mathbf{x}) \forall \mathbf{x} \in E
$$

b) The function $g_{t}$ is continuously differentiable on $\mathrm{E}$ with Lipschitz gradient given by

$$
\nabla g_{t}(x)=\frac{1}{t}\left(I-\operatorname{prox}_{\mathrm{t}}(\mathrm{g})(\mathbf{x})\right) \quad \forall x \in E
$$

c) In particular if $\mathrm{g}=\delta_{\mathrm{c}}$, the indicator of a closed convex set $\mathrm{C} \in \mathrm{E}$, then $\operatorname{prox}_{\mathrm{t}}(\mathrm{g})(\mathbf{x})=(I+\partial g)^{-1}=P_{c}$, the Euclidean projection operator on $\mathrm{C}$ and

$$
g_{t}(\mathbf{x})=\left\|P_{c}(\mathbf{x})-\mathbf{x}\right\|^{2}
$$




\section{PROPOSED WORK}

According to converge of Moreau proximal mapping [6] each IST iterates for solving (4) is given by

$$
\mathbf{x}_{\mathbf{k}+\mathbf{1}}=\boldsymbol{\Psi}_{\mathbf{\tau} \phi}\left(\mathbf{x}_{\mathbf{t}}-\frac{1}{\gamma} \mathbf{A}^{H}\left(A \mathbf{x}_{\mathbf{k}}-y\right)\right)
$$

where $1 / \gamma$ is a step size. Note that the term $\mathbf{A}^{H}\left(\mathbf{A x}_{\mathbf{k}}-y\right)$ is the gradient of the data-fidelity term. It has been proved that if $\gamma>\|\boldsymbol{A}\|_{2}^{2} / 2$ and $\phi$ is convex the algorithm converges to the solution of (4) [8].Further it was proved that IST quite slow and whent is very small and A is ill-conditioned [9].This observation leads to develop the proposed work called Improved Iterative Shrinkage Thresholding algorithm (IISTA).

\subsection{IST Algorithm Derivation}

The equation (5) solves as follows. Fix any scalar $t>0$, then $\mathbf{x}^{*}$ solves the convex minimization problem if and only if the following statements hold:

$$
\begin{array}{cccc}
0 & \epsilon & \mathrm{t} \nabla f\left(\mathbf{x}^{*}\right)+\mathrm{t} \partial g\left(\mathbf{x}^{*}\right) \\
0 & \epsilon & \mathrm{t} \nabla f\left(\mathbf{x}^{*}\right)-\mathbf{x}^{*}+\mathbf{x}^{*}+\mathrm{t} \partial g\left(\mathbf{x}^{*}\right) \\
(I+\partial g)^{-1}\left(\mathbf{x}^{*}\right) & \epsilon & (\mathrm{I}-\mathrm{t} \nabla f)\left(\mathbf{x}^{*}\right) \\
\mathbf{x}^{*}= & (I+\partial g)^{-1}(\mathrm{I}-\mathrm{t} \nabla f)\left(\mathbf{x}^{*}\right)
\end{array}
$$

The equation (11) calls for the fixed point iterative scheme:

$$
\begin{gathered}
\mathbf{x}_{\mathbf{0}} \in E, \quad \mathbf{x}_{\mathbf{k}}=(I+\partial g)^{-1}\left(\mathrm{I}-t_{k} \nabla f\right)\left(\mathbf{x}_{\mathbf{k}-\mathbf{1}}\right), \quad\left(t_{k}>0\right) \\
\mathbf{x}_{\mathbf{k}} \quad=\operatorname{prox}_{t_{k}}(\mathbf{g})\left(\mathrm{I}-t_{k} \nabla f\right)\left(\mathbf{x}_{\mathbf{k}-\mathbf{1}}\right) \\
=\arg \min _{\mathbf{x} \in \mathbf{E}}\left\{\frac{L}{2}\left\|\mathbf{x}-\left(\mathbf{x}_{\mathbf{k}-\mathbf{1}}-t_{k} \nabla f\left(\mathbf{x}_{\mathbf{k}-\mathbf{1}}\right)\right)\right\|^{2}+g(x)\right\}
\end{gathered}
$$

when $\mathrm{g}(\mathbf{x})=\|\mathbf{x}\|_{1}$ the equation reduces to

$$
\mathbf{x}_{\mathbf{k}}=\Psi_{\boldsymbol{\tau} \phi}\left(\mathbf{x}_{\mathbf{k}-\mathbf{1}}-t_{k} \nabla f\left(\mathbf{x}_{\mathbf{k}-\mathbf{1}}\right)\right)
$$

Equation (15) is called IST algorithm [15], where $\boldsymbol{\psi}_{\tau \phi}: \mathbf{E} \rightarrow \mathbf{E}$ is a shrinkage operator defined by $\psi_{\tau \phi}=\left(\left|x_{\mathrm{i}}\right|-\tau\right)_{+} \operatorname{sign}\left(x_{i}\right)$. A typical condition ensuring the convergence of the sequence $\mathbf{x}_{\mathbf{k}}$ produced by IST algorithm is to require $t_{k} \in(0,2 / L(f))$.

\subsection{Improved IST Algorithm}

1. Input an upper bound $L \geq L(f)$ on the Lipschitz constant of $\nabla f$

2. Take $\mathbf{y}_{\mathbf{1}}=\mathbf{x}_{\mathbf{0}} \in \mathbf{E}, \mathbf{t}_{\mathbf{1}}=\mathbf{1}$

3. For $\mathrm{k} \geq 1$. Compute

$$
\begin{gathered}
\mathrm{X}_{\mathrm{k}}=\boldsymbol{p}_{L}\left(\mathrm{y}_{\mathrm{k}}\right) \\
\mathrm{t}_{\mathrm{k}}=\frac{1+\sqrt{1+4 \mathrm{t}_{\mathrm{k}}^{2}}}{2} \\
\mathrm{y}_{\mathrm{k}+1}=\mathrm{x}_{\mathrm{k}}+\left(\frac{t_{k}-1}{t_{k+1}}\right)\left(\mathbf{X}_{\mathrm{k}} \mathbf{X}_{\mathrm{k}-1}\right)
\end{gathered}
$$

Where $\mathrm{p}_{\mathrm{L}}(\mathrm{y})=\operatorname{prox}_{1 / L}(\mathrm{~g})\left(\mathrm{y}-\frac{1}{\mathrm{~L}} \nabla f(y)\right)$

$$
=\arg \min _{\mathbf{x} \in \mathbf{E}}\left\{\frac{L}{2}\left\|\mathbf{x}-\left(\mathbf{y}-\frac{1}{L} \nabla f(\mathbf{y})\right)\right\|^{2}+\mathrm{g}(\mathbf{x})\right\}
$$


This proposed algorithm is called Improved Iterative Shrinkage Thresholding algorithm (IISTA). Each iterate of IISTA depends on the previous two iterate and not only on the last iterate as in ISTA. The operator depends $p_{L}$ uses two previous iterates $\left(\mathbf{X}_{\mathrm{k}}, \mathbf{X}_{\mathrm{k}-1}\right)$ as a linear combination.

The rate of convergence of IISTA is $O\left(1 / \mathrm{k}^{2}\right)$ while it is $\mathrm{O}(1 / \mathrm{k})$ in ISTA.

\section{EXPERIMENTAL RESULTS}

The Experiments are carried with MATLAB R2010a.This work employs total variation as a regulaizer of image restoration problem. The observation shows that objective function monotonically decreasing as the number of iteration increases. The Iterative Signal-to-Noise Ratio (ISNR) increases when the number of iteration increases. The number of iteration required by IISTA for restoration is less than that of ISTA. Hence proposed algorithm is better than ISTA. When the number of iteration increases then the value of objective function decreases.

Experiments are performed using blur size $4 \times 4$ and 9x9. The increase of blur size shows that increase of iteration for restoration.

The variation of different parameters in this studying is shown in Table I to IV.

The results of restoration of ISTA and IISTA are shown in Figures 1, 2 and 3.

\section{CONCLUSION}

The experiments show that IISTA performance is better than that of ISTA in terms of number of iterations required for restoration. The rate convergence of IISTA is more than that of ISTA. When blur size increases the number of iteration also increases. The future study may be using different regulaizer for restoration. The experiment was carried by using total variation.

Table I Variations of different parameters with iterations using ISTA blur size $4 \times 4$

\begin{tabular}{|c|c|c|c|c|}
\hline $\begin{array}{c}\text { Number of } \\
\text { Iterations }\end{array}$ & $\begin{array}{c}\text { Objective function } \\
\left(\mathbf{1 0}^{\mathbf{4}}\right)\end{array}$ & Criterion & ISNR & $\begin{array}{c}\text { CPU time } \\
\text { Seconds }\end{array}$ \\
\hline 0500 & 1.37235 & 0.8517 & 6.794 & 097.48 \\
\hline 1000 & 1.34262 & 0.3016 & 7.155 & 187.41 \\
\hline 1500 & 1.32274 & 0.1433 & 7.446 & 302.91 \\
\hline 2000 & 1.32123 & 0.0700 & 7.652 & 398.38 \\
\hline 2500 & 1.31348 & 0.0441 & 7.722 & 505.04 \\
\hline 3000 & 1.31317 & 0.0242 & 7.793 & 584.27 \\
\hline
\end{tabular}

Table II Variations of different parameters with iterations using IISTA blur size $4 \times 4$

\begin{tabular}{|c|c|c|c|c|}
\hline $\begin{array}{c}\text { Number of } \\
\text { iterations }\end{array}$ & $\begin{array}{c}\text { Objective function } \\
\mathbf{( 1 0}^{\mathbf{4}} \mathbf{)}\end{array}$ & Criterion & ISNR & $\begin{array}{c}\text { CPU time } \\
\text { Seconds }\end{array}$ \\
\hline 050 & 1.39000 & 17.74000 & 6.649 & 11.56 \\
\hline 100 & 1.33174 & 4.20200 & 7.478 & 19.59 \\
\hline 150 & 1.31601 & 0.60700 & 7.903 & 30.72 \\
\hline 200 & 1.31479 & 0.09780 & 7.968 & 38.90 \\
\hline 250 & 1.30899 & 0.08699 & 7.965 & 53.16 \\
\hline 300 & 1.30114 & 0.07359 & 8.080 & 57.87 \\
\hline
\end{tabular}


A. K. Kumaresh

Table III Variation of different parameters using ISTA Blur size 9x9

\begin{tabular}{|c|c|c|c|c|}
\hline Number of iterations & $\begin{array}{c}\text { Objective function } \\
\mathbf{( 1 0}^{\mathbf{3}} \mathbf{)}\end{array}$ & Criterion & ISNR & $\begin{array}{c}\text { CPU time } \\
\text { Seconds }\end{array}$ \\
\hline 1000 & 9.24413 & 0.4265 & 6.679 & 184.12 \\
\hline 2000 & 8.94820 & 0.1473 & 7.167 & 364.27 \\
\hline 3000 & 8.89792 & 0.0762 & 7.504 & 537.14 \\
\hline 4000 & 8.87524 & 0.0459 & 7.796 & 726.74 \\
\hline 5000 & 8.79952 & $0 . ; 032$ & 7.947 & 932.47 \\
\hline
\end{tabular}

Table IV Variations of different parameters using IISTA blur size 9x9

\begin{tabular}{|c|c|c|c|c|}
\hline $\begin{array}{c}\text { Number of } \\
\text { Iterations }\end{array}$ & $\begin{array}{c}\text { Objective function } \\
\left(\mathbf{( 1 0}^{\mathbf{3}} \mathbf{)}\right.\end{array}$ & Criterion & ISNR & $\begin{array}{c}\text { CPU time } \\
\text { Seconds }\end{array}$ \\
\hline 100 & 9.16054 & 5.900 & 6.974 & 20.95 \\
\hline 150 & 8.93014 & 2.846 & 7.589 & 28.74 \\
\hline 200 & 8.85125 & 1.789 & 8.064 & 38.35 \\
\hline 250 & 8.89091 & 0.800 & 8.415 & 52.08 \\
\hline 300 & 8.81801 & 0.331 & 8.633 & 59.64 \\
\hline 400 & 8.72853 & 0.053 & 8.786 & 84.01 \\
\hline 500 & 8.77176 & 0.036 & 8.846 & 106.54 \\
\hline
\end{tabular}

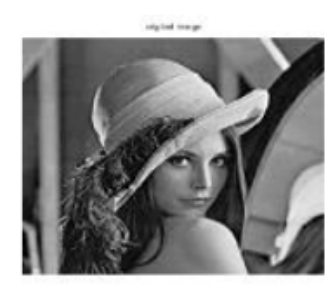

(A)

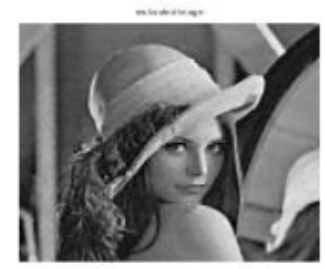

(c)

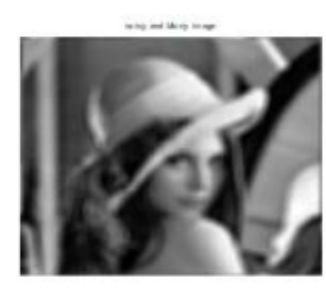

(B)

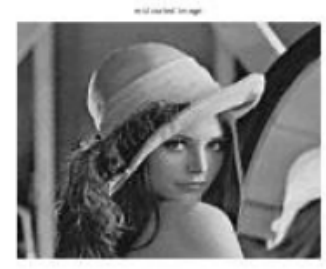

(d)

Figure 1 A) original Image B) noisy and blurry Image blur size $4 \times 4$

C) Restoration using ISTA ISNR $=7.155$ number of iterations $=1000$

D) Restoration using IISTA ISNR $=8.004$ number of iterations $=300$ 
Image Restoration by Improved Iterative Shrinkage Thresholding Algorithm

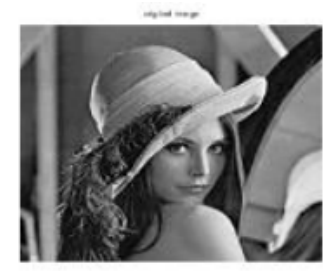

(A)

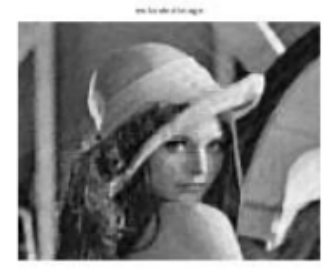

(c)

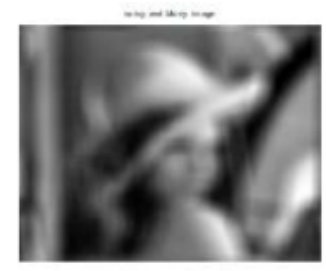

(b)

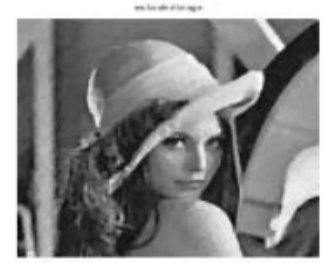

(d)

Figure 2 A) Original Image B) noisy and blurry Image blur size 9x9

C) Restoration using ISTA ISNR $=7.947$ number of iterations $=5000$

D) Restoration using IISTA ISNR $=8.846$ number of iterations $=500$
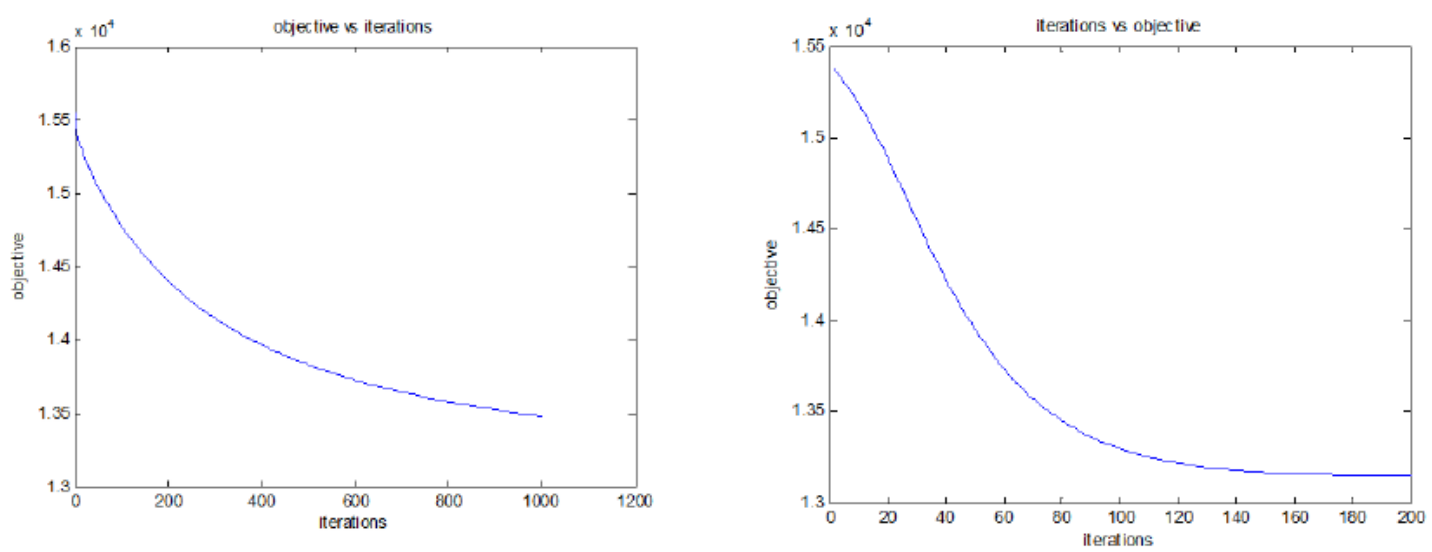

Figure 3 variation of objection function and iterations with ISTA and IISTA

\section{REFERENCES}

[1] Digital Image Processing, by R.C.Gonzalez and R.E.Woods, Addision Wesley An imprint of Pearson Education 2013

[2] "Fundamentals of Digital Image Processing", by A.K.Jain 2014

[3] Digital Image Processing Algorithmic Approach”, by Madhuri A.Joshi, PHI Learning Private Limited 2011

[4] M.Figueiredoand R.Nowak, "An EM Algorithm for wavelet based image restoration”,IEEE Trans. Image Process., Vol 12.no 8 ,pp 906-916,Aug 2003

[5] D.Hunter and K.Lange,"A tutorialon MM algorithms", Amer.Statist., vol 58, pp 30-37, 2004.

[6] I.Daubechies, M.D.Friese and C.D.Mol, "An Iterative thresholding algorithm For linear inverse problems with sparsity constraint", Commu.Pure Appl.Math. Vol 57,pp 1413-1457, 2004 


\section{A. K. Kumaresh}

[7] M.Figueiredoand R.Nowak,"A bound optimization approach to waveler based image Deconvolution “, in Proc. IEEE Int. Conf.Image Processing, Genova, Italy, volII, pp 782785

[8] P.Combettes and V.Wajs, "Signal Recovery by Proximal forward backward Splitting", SIAM J.Multimedia Model Sim., vol 4,pp 1168-1200,2005

[9] T.Hale ,W.Yin and Y.Zhang, "A Fixed Point Continuation Method for $l_{1}$ Regularized Minimization with Applications to Compressed Sensing TR07-07”, TX.Dept. Computat. Appl.Math., Rice Univ., 2007

[10] M.Elad P.Milanfar and R.Rubinstein, "An Analysis Versus Synthesis in signal priors", Inv. Probl. Vol 23, pp. 947-968, 2007

[11] T.Chan S. Esedoglu F Park and A Yip, "Recent developments in total variation Image Restoration", in Handbook of mathematical models in computer vision N.Paragios, Y.Chen and O.Faugeras, Eds New York : Springer -Verlag, 2005

[12] L.Rudin, S.Osher and E.Fatemi, "Non-linear total variation based noise removal algorithms', Physica D vol 60, pp 259-268, 1992

[13] Real Analysis H.L.Royden and P.M.Fitzpatrick Fourth Edition PHI Learning Private Limited 2012

[14] Fast Gradient -Based Algorithms for Constrained Total Variation Image Denoising and Deblurring problems “, Amir Beck and Mare Teboulle May 29, 2009

[15] "Fast Image Recovery using variable splitting and constrained optimization ", M.V.Afonso,J.M.Bioucas-Dias, A.T.Figueiredo. IEEE Transactions on Image Processing.vol 19, No 9,Sep 2010

[16] Digital Image Processing Using MATLAB by R.C.Gonzalez R.E.Woods and S.L.Eddins Mc Graw Hill Education. Second Edition 2017 